\title{
Mechanisms of tumour escape from immune surveillance
}

\author{
Urszula Lisiecka, Krzysztof Kostro \\ ${ }^{1}$ Department of Epizootiology and Clinic of Infectious Diseases, \\ Faculty of Veterinary Medicine \\ University of Life Sciences, 20-950 Lublin, Poland \\ urszula.lisiecka@up.lublin.pl
}

Received: April 13, 2016

Accepted: November 7, 2016

\begin{abstract}
The progressive growth and spread of tumour cells in the form of metastases requires an interaction of healthy host cells, such as endothelial cells, fibroblasts, and other cells of mesenchymal origin with immune cells taking part in innate and adaptive responses within the tumour lesion and entire body. The host cells interact with tumour cells to create a dynamic tumour microenvironment, in which healthy cells can both positively and negatively influence the growth and spread of the tumour. The balance of cellular homeostasis and the effect of substances they secrete on the tumour microenvironment determine whether the tumour has a tendency to grow or disappear, and whether the cells remain within the lesion or are capable of metastasis to other regions of the body. Intercellular interactions also determine the tumour's susceptibility to radiation or other types of cancer treatment. They may also be a rational explanation for differences in treatment outcomes, in which some metastases regress and others progress in response to the same treatment method.
\end{abstract}

Keywords: cancer immunology, immune surveillance, immunosuppression, tumour escape, regulatory T lymphocytes.

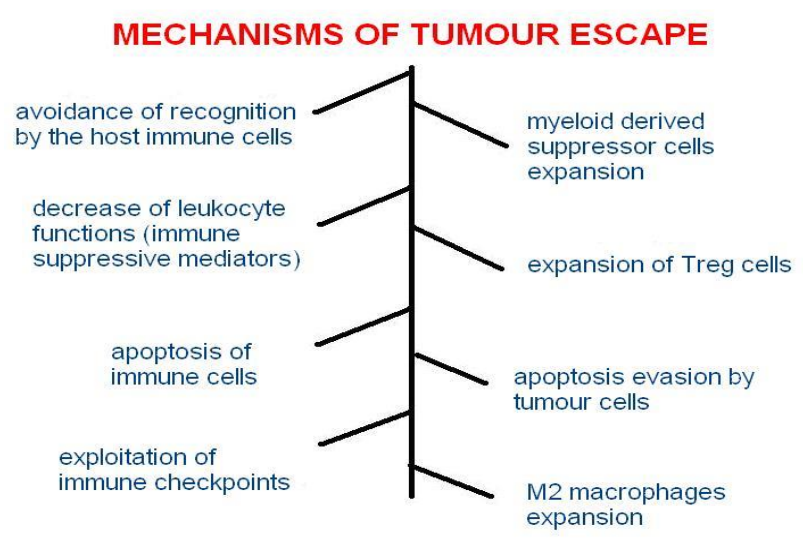

Fig. 1. Major mechanisms of tumour escape from immune surveillance.

In the process of carcinogenesis, despite the development of a specific immune response, spontaneous removal of the tumour by immune mechanisms rarely occurs. Attempts to apply immunotherapy to strengthen the natural tumourspecific immune response also have limited clinical application. This is mainly because cancer cells have a variety of strategies to escape from immune surveillance (53). Escape mechanisms of tumour cells include avoidance of recognition and modulation of the immune functions of effector cells, usually leading to immunosuppression, which facilitates tumour progression and metastasis $(15,26)$.

Avoidance of recognition of tumour cells by the host immune system. Tumour cells, particularly in malignant tumours, often display low immunogenicity, as they are characterised by low or no expression of MHC (major histocompatibility complex) molecules, particularly MHC I, making them difficult to recognise and preventing the initiation of apoptosis, which involves cytotoxic Tc lymphocytes $(37,48)$. Although tumour cells exhibiting low or no expression of MHC I are susceptible to the action of natural killer (NK) cells, they are not destroyed because NK cells have no immunological memory, and there may be too few of 
them in relation to the tumour cells $(24,37)$. Many tumour cells, however, exhibit normal MHC I expression, and therefore are not recognised by NK lymphocytes. They are also unrecognised by cytotoxic $\mathrm{T}$ lymphocytes, because tumour cells often lack tumour-associated antigens (TAA) (38). Furthermore, costimulatory molecules such as CD80 or CD86 are often absent from the surface of tumour cells, which makes activation of $\mathrm{T}$ lymphocytes impossible (37, 46). Other molecules whose expression may be downregulated in tumour cells are latent membrane protein 2 (LMP2), latent membrane protein 7 (LMP7), transporter associated with antigen processing protein (TAP protein), and tapasin $(17,23,32)$. Most tumour antigens that have been identified are proteins which are also found on other cells in the body, leading to the phenomenon of immune tolerance to tumour antigens (24).

Avoidance of recognition by immune cells is also associated with the phenomena of immunodominance and immunoselection. The tumour cell has numerous epitopes which bind directly to soluble antibodies or receptors on the surface of immune cells. The phenomenon of immunodominance is linked to the fact that the parental tumour cells possess dominant antigens and the immune response is directed against these antigens. An important factor determining whether a given antigen will display immunodominance over others is the speed with which it induces an immune response in comparison with other antigens (8). $\mathrm{T}$ cells with cytolytic properties usually react with a particular tumourspecific antigen (TSA) and a single immunodominant epitope of this antigen. During tumour development, dominant antigens may disappear due to immunoselection. As a result of this process, a new hierarchy is established among the remaining antigens whereby one of them becomes the new immunodominant determinant. However, before an effective immune response to the new antigen develops, numerous new tumour cells appear which lose this antigen. These new antigenically modified cells are not recognised by effector immune cells and evade the destruction process. New mutations (translocations or point mutations) cause new antigen epitopes to appear on the surface of tumour cells, and these are often able to induce a much stronger immune response than the primary antigen. However, a study on mice demonstrated that the existing immune response to the antigen initially present on tumour cells inhibits the immune response to new antigens $(3,8,42)$.

Immunoregulatory effect of tumour cells on leukocyte functions. Tumour cells secrete a wide range of substances known as tumour-derived soluble factors (TDSFs), which decrease or inhibit the local immune response. These include cytokines such as interleukin 6 (IL-6) and interleukin 10 (IL-10), as well as tumour growth factor TGF- $\beta$ and vascular endothelial growth factor VEGF. Their activity gradually spreads from the tumour environment to adjacent lymphatic organs and peripheral blood vessels $(24,25,37)$. Moreover, tumours often have a potential of shifting the balance from Th1 to Th2. Th1 type of immune response is characterised by cytokines which are produced by Th1 $\mathrm{CD}^{+}$ lymphocytes, the main being interferon- $\gamma$ (IFN $\gamma$ ), which is the chief proinflammatory factor. By contrast, Th2 cytokines, such as interleukin 4 (IL-4), interleukin 5 (IL-5), interleukin 10 (IL-10), and interleukin 13 (IL-13) tend to produce the antiinflammatory response. Th2 anti-inflammatory response counteracts the Th1 mediated antitumour inflammatory processes. Neoplastic cells create this Th2 anti-inflammatory tumour milieu by secreting chemokines with chemotactic properties for immunosuppressive regulatory $\mathrm{T}$ lymphocytes and subpopulations of Th2 lymphocytes associated with humoral response, so these subpopulations are attracted to the tumour site. In contrast, cytotoxic Tc lymphocytes, which are capable of killing tumour cells, usually have no receptors for these chemokines, so they are not directed towards the tumour and are not able to perform their function at the site affected by the neoplastic process $(18,32)$. Furthermore, cytokines and chemokines secrete soluble receptors inducing apoptosis of effector cells, such as human leukocyte antigen 1 (HLA-1), as well as other soluble receptors which bind to surface structures of cytotoxic $\mathrm{T}$ lymphocytes and NK cells and block their receptors, thereby preventing recognition of tumour cells and activation of these lymphocytes. The presence of these substances decreases the activation of effector cells. One such receptor is soluble MHC class I polypeptide-related sequence A (sMIC-A), which binds to the lectin-like activation receptor CD314 (NKG2D-type integral membrane protein) on the surface of $\mathrm{T}$ lymphocytes and $\mathrm{NK}$ cells, thereby preventing them from binding the MIC-A protein. The MIC-A protein is a surface molecule related to $\mathrm{MHC}$ I molecules. It is expressed on the surface of tumour cells under stress conditions or during viral infections. Beside MIC-A, other ligands of this type are UL16 binding proteins (ULBPs), which are probably produced by tumour cells and block receptor CD316 (NKG2D), (37, 38).

Depriving $\mathrm{T}$ cells of the amino acids such as tryptophan and arginine impairs their function and proliferation capacity (18). Changes in amino acid metabolism and their degradation are another mechanism by which tumours induce immunosuppression - both local, within the lesion itself, and systemic. Myeloid-derived suppressor cells (MDSC) are probably responsible for these processes. Tumour development is accompanied by enhanced expression of the enzyme indoleamine 2,3dioxygenase (IDO), whose presence causes a local reduction in tryptophan and leads to the production of 
kynurenine, a catabolite causing anergy and death of $\mathrm{T}$ lymphocytes and NK cells. Through the involvement of immature dendritic cells (iDCs), IDO is presented to virgin $\mathrm{T}$ lymphocytes, which can transform into regulatory $\mathrm{T}$ cells and cause systemic anergy for tumour-associated antigens (TAA). A similar enzyme that exerts immunosuppressive activity is arginase, which breaks down L-arginine to ornithine. This process promotes tumour growth and reduces the activity of effector $\mathrm{T}$ lymphocytes, probably by blocking the $\zeta$-chain of receptor CD3 $(3,38)$.

Myeloid cells and tumour development. Suppressive cells outside the T lymphocyte system are sometimes collectively referred to as myeloid-derived suppressor cells (MDSCs). They are a heterogeneous population of cells consisting of immature cells of myeloid lineage, i.e. macrophages, granulocytes, dendritic cells, and other cells in early stages of differentiation. Their presence has been detected in the spleen, bone marrow, and peripheral blood, as well as in the tumour microenvironment $(30,54)$. Formation of MDSCs is induced by substances secreted by the tumour, which may be granulocytemacrophage colony-stimulating factor (GM-CSF), vascular endothelial growth factor (VEGF), colonystimulating factor 1 (CSF-1), prostaglandin E2 (PGE2), IL-6, or IL-10 (49). Chronic inflammation accompanying cancer may also be a factor conducive to the formation of MDSCs. VEGF produced within the tumour is a strong chemoattractant which increases recruitment of immature myeloid cells (iMC) from the bone marrow to the peripheral blood vessels, from which they are attracted to the tumour site by chemokines. These cells undergo functional and biochemical modulation in the tumour environment, becoming tumour-associated dendritic cells (TiDC) and tumour-associated macrophages (TAM). An increased number of immature MDSCs have been observed in the peripheral blood and lymph nodes of human patients with breast, head, neck, and lung cancers. Immature TiDC not only do not initiate an antitumour response involving $\mathrm{T}$ lymphocytes, but are actually inhibitors of such a response, causing a state of peripheral tolerance for tumour cells (26).

MDSCs migrate from the tumour environment to the lymph nodes, spleen, and peripheral vessels, where they inhibit activation of tumour-specific $\mathrm{T}$ lymphocytes due to the elevated level of reactive oxygen species (ROS). In addition, MDSCs inhibit the immune response to tumour-associated antigens (TAA) and also block the functioning of antitumour $\mathrm{T}$ lymphocytes by producing arginase-1, an enzyme breaking down arginine from the tumour environment, which impairs signal transmission in these lymphocytes. Furthermore, MDSCs produce nitric oxide synthase (NOS) leading to the generation of nitric oxide, whose mechanism of immunosuppressive action against effector immune cells has yet to be fully clarified. They may also induce accumulation of Treg $\mathrm{CD}^{+}{ }^{+} \mathrm{Foxp}^{+}$regulatory lymphocytes, which have suppressive properties $(10,27,30,54)$.

Immunosuppressive properties of $T$ regulatory (Treg) lymphocytes and their influence on tumour development. Another significant mechanism conducive to tumour progression is inhibition of the cellular immune response through an increase in the percentage of regulatory $\mathrm{T}$ (Treg) cells as a result of the neoplastic process. These cells are a separate phenotypically heterogeneous group of lymphocytes responsible for regulating immune functions. Treg cells have been detected and characterised in mice, rats, humans, baboons, macaques, chimpanzees, cats, dogs, and pigs. There is also convincing evidence of Treg cell populations in cows, sheep, and horses. In humans, rodents, and cats they account for about $5 \%-10 \%$ of the population of peripheral $\mathrm{CD}^{+}$lymphocytes. A few subpopulations of regulatory cells have been distinguished and one of the most important in terms of neoplastic processes is subpopulation of $\mathrm{CD} 4{ }^{+} \mathrm{CD} 25^{+} \mathrm{T}$ lymphocytes, exhibiting expression of the intracellular forkhead box P3 (Foxp3) protein $(14,20)$. A characteristic trait of Treg cells is constant high expression of the $\alpha$ subunit of the IL-2 receptor (CD25), in contrast with activated $\mathrm{CD}^{+}$and $\mathrm{CD}^{+}$lymphocytes, which exhibit lower transient expression of this marker $(33,39)$.

Initially, subpopulations of regulatory lymphocytes were identified using antibodies against CD4 and CD25 molecules. It was later established that Treg cells are exclusively those lymphocytes which are characterised by a high level of CD25 expression (phenotype $\mathrm{CD} 4^{+} \mathrm{CD} 25^{\text {high }}$ ). Currently, the most specific marker of regulatory lymphocytes in humans, rodents, and $\operatorname{dogs}$ is considered to be the intracellular protein Foxp3, which plays a fundamental role in Treg cell function and is necessary for them to differentiate properly $(5,20,33$, 36).

On the basis of their origin, Treg cells are divided into two subgroups: cells that arise within the thymus, known as natural Treg (nTreg) cells and cells induced in the periphery-induced Treg (iTreg) cells also known as adaptive Treg (aTreg) cells (33, 53). The activity of regulatory $\mathrm{T}$ lymphocytes involves regulation of the immune response at the peripheral level. They are part of the mechanism inhibiting selectively activated cells with autoreactive properties. This prevents the development of autoimmune processes caused by an excessive immune response to self-antigens.

Regulatory cells have an important function in tumour immunology, allogeneic transplants, pathogenesis of allergic diseases, and regulation of certain antimicrobial defence mechanisms. Under certain conditions, regulatory $\mathrm{T}$ lymphocytes may exert a negative effect, inhibiting the antitumour immune response $(5,52)$. 


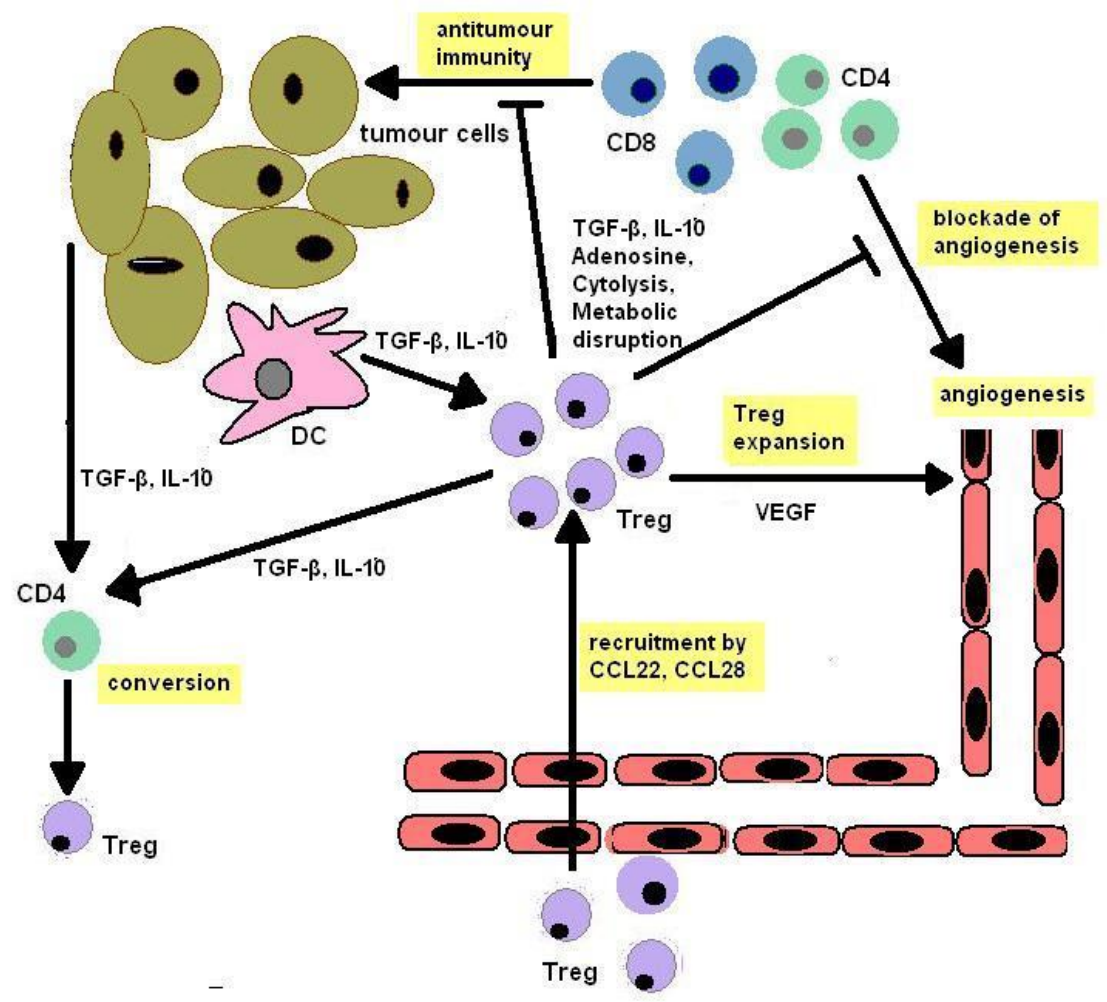

Fig. 2. The role of Treg cells in tumour progression. Adopted and reproduced from Faciabenne et al. (12)

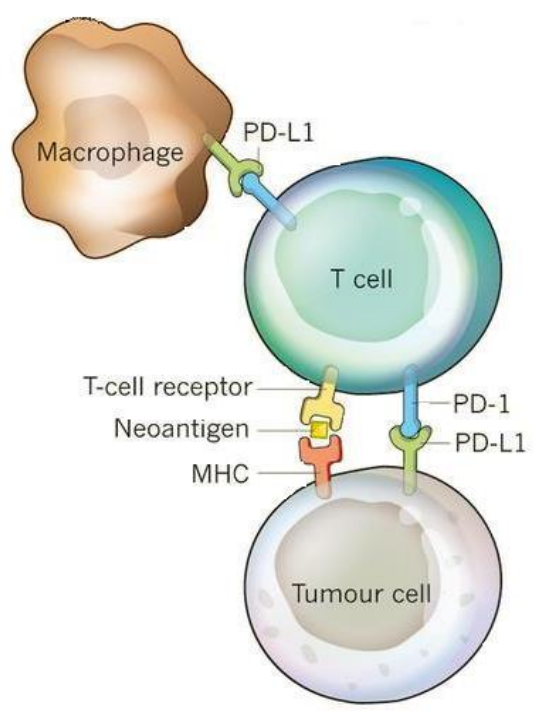

Inhibited antitumour immunity

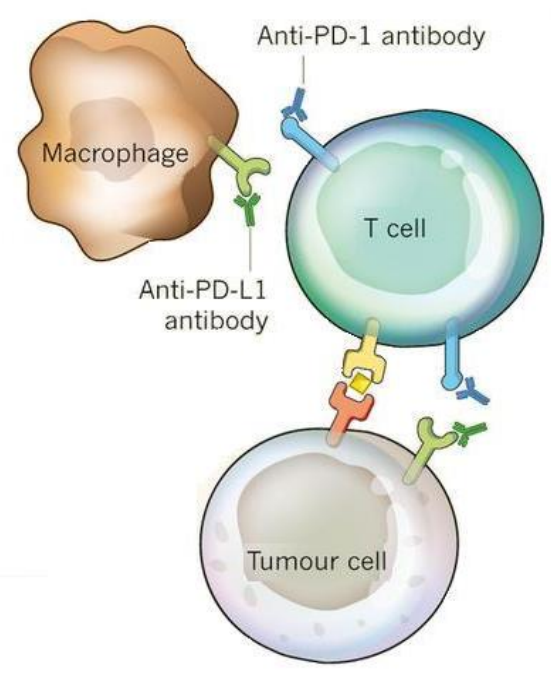

Enhanced antitumour immunity

Fig. 3. Checkpoint PD-1 blockade activates antitumour immunity. Adopted and reproduced from Wolchok and Chan (50)

Elevated numbers of Treg cells have been observed within tumour mass, in close proximity to the tumour, in tumour draining lymph nodes, and in peripheral blood of patients with diverse types of cancers. There are several mechanisms of Treg cell accumulation in tumours. Treg cells may be recruited to tumours by hypoxia-induced chemokines produced by tumour cells, such as C-C motif chemokine ligand
28 (CCL28) and C-C motif chemokine ligand 22 (CL22). A second mechanism is intensive proliferation of Treg cells within tumour mass. A proof confirming this phenomenon could be the presence of Ki-67 protein in tumour infiltrating Treg cells. Ki-67 protein is a nuclear antigen, expressed by proliferating cells. Moreover, subpopulation of Treg cells can be expanded de-novo by inversion of CD4+ 
naive precursors within the tumour microenvironment, with the presence of TGF $\beta$ and IL-10 $(4,35)$.

In particular, Treg lymphocytes arising in the periphery play a role in active suppression of the antitumour effector activity of $\mathrm{CD}^{+}$and $\mathrm{CD}^{+}$ lymphocytes by producing specific cytokines, such as IL-10, IL-4, and IL-13 (47, 53). Regulatory lymphocytes are capable of inhibiting proliferation of $\mathrm{CD} 4^{+} \mathrm{CD} 25^{-} \mathrm{T}$ lymphocytes and blocking them from secreting cytokines. Moreover, they can inhibit subpopulations of B lymphocytes, NK cells, and even dendritic cells $(28,53)$. They also inhibit $\mathrm{CD}^{+}{ }_{-}^{-}$ dependent inflammatory reactions and eliminate $\mathrm{CD}^{+}$ cytotoxicity against tumour cells. Beside their effect on $\mathrm{T}$ lymphocytes, regulatory $\mathrm{T}$ cells inhibit the proliferation and production of antibodies by $\mathrm{B}$ lymphocytes of $\mathrm{CD} 19^{+}$phenotype (53). The effect of regulatory lymphocytes on innate immune cells has been demonstrated. An example is inhibition of the cytotoxicity of $\mathrm{CD} 3{ }^{-} \mathrm{CD} 56^{+} \mathrm{NK}$ cells and stimulation of differentiation of monocytes into alternatively activated tumour-associated macrophages (TAM) contributing to the development of tumour tissue (47). Treg cells also stimulate tumour angiogenesis directly by producing VEGF and indirectly by blocking effector-cell-derived angiostatic cytokines, such as IFN- $\gamma$ and C-X-C motif chemokine 10 (CXCL-10) (4, $12,34,35)$.

Increased numbers or frequency of Treg cells in a variety of cancers is correlated with poor prognosis. However, in some types of cancers increased levels of Treg cells may be connected with favourable prognosis, which might relate to the ability of these cells to reduce inflammation. Inflammation is one of the crucial factors of tumour progression in many tissues, because it increases proliferation of tumour cells, angiogenesis, and metastasis. It is highly probable that the disease outcome is dependent on the balance between $\mathrm{T}$ effector cells and $\mathrm{T}$ regulatory cells $(9,13,35,4043)$.

Apoptosis of immunocompetent cells. The cells of some tumours are capable of inducing apoptosis in immune effector cells. Melanoma cells can release exosomes containing Fas ligand (FasL), which interacts with the Fas molecule on the surface of lymphocytes leading to their death by apoptosis. Exosomes are small particles of 80-200 nm in diameter, whose potential role is to transmit signals among antigen-presenting cells. The Fas molecule belongs to the family of death receptors, which are involved in initiating a cascade of apoptotic signals in T cells $(15,19)$. In the initial stages of the neoplastic process, tumour cells secrete exosomes containing FasL on their surface and cause apoptosis of $\mathrm{T}$ lymphocytes, either directly or indirectly through dendritic cells binding exosomes secreted by the tumour (19). Some studies indicate that FasL can be released not only by tumour cells but also by activated NK cells themselves, which leads to their own apoptosis. It is likely that each time an NK cell is killing a tumour cell; it also activates its own apoptosis process and dies (37). Apoptosis mechanisms in immunocompetent cells can also be induced by immunosuppressive cytokines located in the tumour microenvironment, such as TGF- $\beta$ and IL10 , by changes in red-ox potential, or by changes in the intracellular calcium or ceramide level (15).

Apoptosis evasion by tumour cells. A condition of maintaining homeostasis in the body is efficient elimination of superfluous cells by apoptosis. Numerous mutations accompanying tumour formation, such as the loss of pro-apoptotic factors like tumour suppressor protein p53 (p53) and Bcl-2 associated X (Bax) protein, or overexpression of antiapoptotic proteins Bcl-2 (B-cell lymphoma 2 protein), or myeloid cell leukaemia 1 (Mcl-1) protein, lead to a blockade of the intrinsic apoptosis pathway, and in consequence prevent effective elimination of neoplastically transformed cells $(29,41)$. Tumour cells produce soluble phosphatidylserine (sP), which interferes with the elimination of apoptotic cells. It blocks phosphatidylserine receptors (PSR) on dendritic cells and macrophages, which cannot bind phosphatidylserine on the surface of apoptotic cells, so that they are not recognised and ingested by phagocytes. The interaction of soluble phosphatidylserine with PSR receptors on the surface of macrophages causes the release of antiinflammatory mediators, such as IL-10, TGF- $\beta$, or prostaglandin $\mathrm{E}_{2}\left(\mathrm{PGE}_{2}\right)$ (26). Some cytokines which have an antiproliferative and pro-apoptotic effect on normal cells remain inactive against tumour cells. This state becomes established as the tumour progresses, because cytokine receptors on the cells of many types of tumour exhibit reduced expression or become modified. This occurs in the case of TGF $\beta$, which may stimulate the growth of tumour cells rather than inhibit it due to modification of the TGF $\beta$ type II receptor present on the surface of these cells. A similar example is the effect of IL-6, which inhibits the growth of melanoma cells in the initial stages, but stimulates proliferation of the tumour in advanced stages and metastases (7). Moreover, it is hypothesised that tumour cells exhibiting high MHC I antigen expression may evade apoptosis by a mechanism associated with an increased level of heat shock protein HSP90 in the cell nucleus (15). Disturbances in the elimination of apoptotic cells lead to the formation of autoantibodies, resulting in a pseudo-autoimmune status, which in turn can initiate the generation of regulatory Treg lymphocytes inhibiting the antitumour response of $\mathrm{T}$ lymphocytes (26).

Immune checkpoints. Under physiological conditions the immunological pathways are functioning properly, which plays a crucial role in regulating immune responses to pathogens and preventing autoimmune reactions. These pathways are 
regulated by immune checkpoints, which under malignant conditions may be used by tumour cells to facilitate their growth and progression. One of these checkpoints is programmed cell death protein 1 (PD-1 protein) also known as cluster of differentiation 279 (CD279), which is a cell surface receptor of $\mathrm{T}$ cells and pro-B cells. PD-1 binds two ligands, PD-L1 and PD-L2. The PD-1 pathway blocks T-cell response at the late effector stage preventing excessive $\mathrm{T}$ cell activation. In order to evade antitumour immune response, tumours can express PD-L1 and PD-L2 ligands. Interactions via PD-1:PD-L1 and PD-1:PDL2 cause chronic T-cells inhibition, which leads to their anergy and decreases immune response to cancer cells. PD-L2 expression on tumour cells has been found to be a negative prognostic factor in some human tumours $(22,31)$. Anti-tumour activity may be enhanced by blocking PD-1 and PD-L1 receptors with specific monoclonal antibodies (50).

Another checkpoint which may be used by tumours to dampen the immune response is cytotoxic T-lymphocyte associated protein 4 (CTLA-4), also known as cluster of differentiation 152 (CD152). CTLA-4 plays an important role in early stages of immune response. It competes with CD28 in binding to B7 ligands on antigen presenting cells (APCs). This prevents further $\mathrm{T}$ cell activation and limits the time of $\mathrm{T}$ cell activity (31). CTLA-4 is constitutively expressed on tumour cells and it behaves as a negative regulator of proliferation and effector function of $\mathrm{T}$ cells $(11,48)$. Another example of immune checkpoint molecule is a surface receptor named glucocorticoid-induced tumour necrosis factor receptor (GITR). This molecule is highly expressed on $\mathrm{T}$ regulatory cells, but it is also present on normal effector T-cells after activation, on tissue-infiltrating macrophages and dendritic cells. GITR interacts with ligand GITRL and these interactions induce production of specific cytokines and chemokines at local inflammatory sites, which enables the control of tissue-infiltrating effector or regulatory cell activation and their migration $(1,44)$. The GITR is responsible for inhibition of the suppressive activity of T-regulatory cells and for enhancement of the survival of T-effector cells. These properties of the GITR enable to enhance the immunity to tumours and viral pathogens, and to exacerbate autoimmune disease. Some human cancers and many tumour cell lines of different histological origin express substantial levels of GITRL (21). Signalling through GITRL downregulates the expression of the immunostimulatory molecules, such as CD40 and CD54. Moreover, GITR-GITRL interaction negatively influences the expression of the adhesion molecule EpCAM (epithelial cell adhesion molecule) and induces production of the immunosuppressive factor TGF $\beta$ by tumour cells. Activated NK cells constitutively express the GITR. In tumour and NK cell cocultures, cytotoxicity, and IFN production was remarkably increased after blocking GITR-GITRL interaction. It could be a proof that expression of GITRL on the surface of tumour cells may lower NK cell antitumour properties (2). Furthermore, one of the immune checkpoints used by cancer cells for immune escape is lymphocyte activation gene 3 (LAG-3) molecule, which is an inhibitory receptor present on immune cells. LAG-3 molecule is the target of various drug development programmes and trials with new treatments of cancer. This protein belongs to immunoglobulin superfamily and was designated as CD223 cluster of differentiation. LAG-3 is expressed on activated $\mathrm{T}, \mathrm{NK}, \mathrm{B}$, and plasmacytoid dendritic cells. LAG-3 binds to MHC class II with higher affinity than $\mathrm{CD} 4$, and negative regulatory role for the LAG3/MHC II interaction has been proven. LAG-3 negatively affects immune cells proliferation, activation, and homeostasis in a similar way to CTLA-4 and PD-1. Additionally, it is involved in suppressive function of Treg cells, and in mitigating dendritic cell differentiation and function. Moreover, it exerts diverse biological effects on the function of $\mathrm{T}$ lymphocytes, such as maintaining CD8+ T-cell tolerance $(16,51)$. Extensive coexpression of PD-1 and LAG-3 on tumour infiltrating CD4 and CD8 $\mathrm{T}$ cells gives evidence for a possible role of these receptors in tumour immune escape. Moreover, combinatorial anti-LAG-3 and anti-PD-1 immunotherapy inhibits tumour growth (51).

M2 macrophages. There is increasing evidence that macrophages may contribute to tumour growth and progression. It is commonly known that tumour lesions are infiltrated by macrophages which are called tumour associated macrophages (TAMs). Macrophages in tumour microenvironment have been discovered to have M1 or M2 phenotype. M1 macrophages stimulate inflammation and secrete high levels of IL-12 and low levels of IL-10, while M2 macrophages decrease inflammation, augment tissue repair, and produce anti-inflammatory cytokines like IL-10 or TGF- $\beta$. The dominance of M2 phenotype macrophages in tumour microenvironment usually correlates with poor prognosis, because M2 macrophages seem to actively promote tumour growth. Macrophages of M2 phenotype are either recruited by tumour cells and infiltrate tumour tissues, or may acquire an M2 phenotype in tumour microenvironment through transformation from M1 to M2 macrophages. Macrophages are recruited to tumour microenvironment from blood-stream monocytes by chemotactic factors such as chemokine C-C motif ligand 2 (CCL2), also referred to as MCP1monocyte chemoattractant protein 1, vascular endothelial growth factor (VEGF), macrophage colony-stimulating factor (M-CSF), and C-X-C motif chemokine 12 (CXCL12), also known as SDF1stromal cell-derived factor 1 , which are released by malignant and stromal tumour cells. Macrophages of M2 phenotype when stimulated by low oxygen 
content of their surroundings produce pro-angiogenic factors which mediate and speed up angiogenesis and promote further tumour growth. These factors include VEGF, tumour necrosis factor- $\alpha$ (TNF- $\alpha$ ), granulocyte macrophage colony-stimulating factor (GM-CSF), IL1, and IL-6. Recent studies revealed that hypoxia (or extremely low oxygen tension), which is an important tumour microenvironmental factor, can induce the activation of nuclear factor- $\kappa \mathrm{B}(\mathrm{NF}-\kappa \mathrm{B})$ in murine as well as in human macrophages. The transcription factor $N F-\kappa B$ is driving the tumour promoting functions of macrophages by regulating the expression of many important genes in TAMs, and influences molecular mechanisms that promote tumourigenesis $(6,45,55)$.

The review presents the most recent data concerning mechanisms of tumour escape from immunological surveillance, which is one of the most important immunological aspects of tumourigenesis. Gaining detailed knowledge about the immune evasion strategies raises hope for developing new, safer and more effective methods of cancer prophylaxis and treatment. It is an especially vital issue because neoplasms are currently one of the most frequent diseases of affluence and they constitute a serious problem both in human and veterinary medicine. Rapidly growing number of animals with oncological problems, observed particularly in the area of urban agglomerations, constitutes significant evidence that there is an urgent need for fundamental understanding of complicated interactions between a neoplasm and the immune system.

Conflict of Interests Statement: The authors declare that there is no conflict of interests regarding the publication of this article.

\section{References}

1. Azuma M.: Role of the glucocorticoid-induced TNFR-related protein (GITR)-GITR ligand pathway in innate and adaptive immunity. Crit Rev Immunol 2010, 30, 547-557.

2. Baltz K.M., Krusch M., Bringmann A., Brossart P., Mayer F., Kloss M., Baessler T., Kumbier I., Peterfi A., Kupka S., Kroeber S., Menzel D, Radsak M.P., Rammensee H.G., Salih H.R.: Cancer immunoediting by GITR (glucocorticoid-induced TNFrelated protein) ligand in humans: NK cell/tumor cell interactions. FASEB J 2007, 21, 2442-2454.

3. Beatty G.L., Gladney W.L.: Immune escape mechanisms as a guide for cancer immunotherapy. Clin Cancer Res 2015, 21, doi: 10.1158/1078-0432.

4. Beyer M., Schultze J.L.: Regulatory T cells: major players in the tumor microenvironment. Curr Pharm Des 2009, 15, 1879-1892.

5. Biller B.J., Elmslie R.E., Burnett R.C., Avery A.C., Dow S.W.: Use of FoxP3 expression to identify regulatory $\mathrm{T}$ cells in healthy dogs and dogs with cancer. Vet Immunol Immunopathol 2007, 116, 69-78.

6. Biswas S.K., Lewis C.E.: NF- $\kappa \mathrm{B}$ as a central regulator of macrophage function in tumors. J Leukoc Biol 2010, 88, 877884.

7. Candolfi M., Curtin J.F., Yagiz K., Assi H., Wibowo M.K., Alzadeh G.E., Foulad D., Muhammad A.K.M.G., Salehi S.,
Keech N., Puntel M., Liu Ch., Sanderson N.R., Kroeger K.M., Dunn R., Martins G., Lowenstein P.R., Castro M.G.: B cells are critical to T-cell-mediated antitumor immunity induced by a combined immune-stimulatory/conditionally cytotoxic therapy for glioblastoma. Neoplasia 2011, 13, 947-960.

8. Cohen A.D., Schaer D.A., Liu C., Li Y., HirschhornCymmerman D., Kim S.C.: Agonist anti-GITR monoclonal antibody induces melanoma tumor immunity in mice by altering regulatory $\mathrm{T}$ cell stability and intra-tumor accumulation. PLoS ONE 2010, 5, e10436. doi:10.1371/journal.pone.0010436.

9. Colotta F., Allavena P., Sica A., Garlanda C., Mantovani A.: Cancer related inflammation, the seventh hallmark of cancer: links to genetic instability. Carcinogenesis 2009, 30, 1073-1081.

10. Condamine T., Ramachandran I., Youn J., Gabrilovich D.I.: Regulation of tumor metastasis by myeloid-derived suppressor cells. Ann Rev Med 2015, 66, 97-110.

11. Contardi E., Palmisano G.L., Tazzari P.L., Martelli A.M., Falà F., Fabbi M., Kato T., Lucarelli E., Donati D., Polito L., Bolognesi A., Ricci F., Salvi S., Gargaglione V., Mantero S., Alberghini M., Ferrara G.B., Pistillo M.P.: CTLA-4 is constitutively expressed on tumor cells and can trigger apoptosis upon ligand interaction. Int J Cancer 2005, 117, 538-550.

12. Facciabene A., Motz G.T., Coukos G.: T-regulatory cells: key players in tumor immune escape and angiogenesis. Cancer Res 2012, 72, 2162-2171.

13. Galon J., Costes A., Sanchez-Cabo F., Kirilovsky A., Mlecnik B., Lagorce-Pagès C., Tosolini M., Camus M., Berger A., Wind P., Zinzindohoué F., Bruneval P., Cugnenc P.H., Trajanoski Z., Fridman W.H., Pagès F.: Type, density, and location of immune cells within human colorectal tumors predict clinical outcome. Science 2006, 313, 1960-1964.

14. Garden O.A., Pinheiro D., Cunninghamb F.: All creatures great and small: regulatory $\mathrm{T}$ cells in mice, humans, dogs and other domestic animal species. Int Immunopharmacol 2011, 11, 576588 .

15. Gastman B.R., Johnson D.E., Whiteside T.L., Rabinowich H.: Tumor-induced apoptosis of $\mathrm{T}$ lymphocytes: elucidation of intracellular apoptotic events. Blood 2000, 95, 2015-2023.

16. Goldberg M.V., Drake C.G.: LAG-3 in cancer immunotherapy. Curr Top Microbiol Immunol 2011, 344, 269-278.

17. Hayashi T., Horiuchi A., Sano K., Hiraoka N., Kasai M., Ichimura T., Sudo T., Tagawa Y., Nishimura R., Ishiko O., Kanai Y., Yaegashi N., Aburatani H., Shiozawa T., Konishi I.: Potential role of LMP2 as tumor-suppressor defines new targets for uterine leiomyosarcoma therapy. Sci Rep 2011, 1, 180, doi: 10.1038/srep00180.

18. Huye L.E., Dotti G.: Designing $T$ cells for cancer immunotherapy. Discov Med 2010, 47, 297-303.

19. Ichim T.E., Zhong Z., Kaushal S., Zheng X., Ren X., Hao X., Joyce J.A., Hanley H.H., Riordan N.H., Koropatnick J., Bogin V., Minev B.R., Min W., Tullis R.H.: Exosomes as a tumor immune escape mechanism: possible therapeutic implications. J Transl Med 2008, 6, 37 http://www.translationalmedicine.com/content/6/1/37.

20. Jagła M., Cichocka-Jarosz E.: Regulatory lymphocytes. Alerg Astma Immun 2007, 12, 22-29.

21. Ji H.B., Liao G., Faubion W.A., Abadia-Molina A.C., Cozzo C., Laroux F.S., Caton A., Terhorst C.: Cutting edge: the natural ligand for glucocorticoid-induced TNF receptor-related protein abrogates regulatory T cell suppression. J Immunol 2004, 172, 5823-5827.

22. Jiabei H., Ying H., Mingming H., Baolan L.: Development of PD-1/PD-L1 pathway in tumor immune microenvironment and treatment for non-small cell lung cancer. Sci Rep 2015, 5, doi:10.1038/srep13110.

23. Johnsen A.K., Templeton D.J., Sy M., Harding C.V.: Deficiency of transporter for antigen presentation (TAP) in tumor cells allows evasion of immune surveillance and increases tumorigenesis. J Immunol 1999, 163, 4224-4231. 
24. Kaufman H.L., Disis M.L.: Immune system versus tumor: shifting the balance in favor of DCs and effective immunity. J Clin Invest 2004, 113, 664-667.

25. Kim P., Armstrong T., Song H., Wolpoe M., Weiss V., Manning E., Huang L., Murata S., Sgouros G., Emens L., Reilly R., Jaffe E.: Antibody association with HER-2/neu-targeted vaccine enhances $\mathrm{CD} 8+\mathrm{T}$ cell responses in mice through $\mathrm{Fc}$-mediated activation of DCs. J Clin Invest 2008, 188, 1700-1711.

26. Kim R., Emi M., Tanabe K.: Cancer immunosuppression and autoimmune disease: beyond immunosuppressive networks for tumour immunity. Immunology 2006, 119, 254-264.

27. Kumar V., Patel S., Tcyganov E., Gabrilovich D.I.: The nature of myeloid derived suppressor cells in the tumor microenvironment. Trends Immunol 2016, 37, 208-220.

28. Lewkowicz P., Lewkowicz N., Tchórzewski H.: CD4 ${ }^{+} \mathrm{CD} 25^{+} \mathrm{T}$ regulatory cells in pathophysiology and therapy of immunological disorders. Postępy Hig Med Dośw 2005, 59, $371-376$.

29. Lopez J., Tait S.W.G.: Mitochondrial apoptosis: killing cancer using the enemy within. Br J Cancer 2015, 112, 957-962.

30. Luczyński W., Krawczuk-Rybak M., Stasiak-Barmuta A.: Myeloid-derived suppressor cells - the new mechanism of immunosuppression in cancer. Postępy Hig Med Dośw 2008, 21, 18-22.

31. Luke J.L., Ott P.A.: PD-1 pathway inhibitors: The next generation of immunotherapy for advanced melanoma. Oncotarget 2015, 6, 3479-3492.

32. Maeda H., Shiraishi A.: TGF-beta contributes to the shift toward Th-2 type responses through direct and IL-10-mediated pathways in tumor-bearing mice. J Immunol 1996, 156, 73-78.

33. Mougiakakos D.: Regulatory T cells in colorectal cancer: from biology to prognostic relevance. Cancers 2011, 3, 1708-1731.

34. Nishikawa H., Sakaguchi S.: Regulatory $\mathrm{T}$ cells in tumor immunity. Int J Cancer 2010, 127, 759-767.

35. Oleinika K., Nibbs R.J., Graham G.J., Fraser A.R.: Suppression, subversion and escape: the role of regulatory $\mathrm{T}$ cells in cancer progression. Clin Exp Immunol 2013, 171, 36-45.

36. Pinheiro D., Singh Y., Grant Ch.R., Appleton R.C., Sacchini F., Walker K.L.R., Chadbourne A.H., Palmer Ch.A., ArmitageChan E., Thompson I., Williamson L., Cunningham F., Garden O.A.: Phenotypic and functional characterization of a CD4+CD25high FOXP3high regulatory T-cell population in the dog. Immunology 2010, 132, 111-122.

37. Poggi A., Massaro A.M., Negrini S., Contini P., Zocchi M.R.: Tumor-induced apoptosis of human IL-2-activated NK cells: role of natural cytotoxicity receptors. J Immunol 2005, 174, 26532660.

38. Poggi A., Zocchi M.R.: Mechanisms of tumor escape: role of tumor microenvironment in inducing apoptosis of cytolytic effector cells. Arch Immunol Ther Exp 2006, 54, 323-333.

39. Ryba M., Myśliwska J.: CD4+CD25+Foxp3+ T lymphocytes: naturally occurring $\mathrm{T}$ regulatory lymphocytes. Pediatr Endocrinol Diabetes Metab 2010, 16, 289-294.

40. Sato E., Olson S.H., Ahn J., Bundy B., Nishikawa H., Qian F., Jungbluth A.A., Frosina D., Gnjatic S., Ambrosone C., Kepner J., Odunsi T., Ritter G., Lele S., Chen Y.T., Ohtani H., Old L.J.,
Odunsi K.: Intraepithelial CD8+ tumor-infiltrating lymphocytes and a high $\mathrm{CD} 8+$ /regulatory $\mathrm{T}$ cell ratio are associated with favorable prognosis in ovarian cancer. Proc Natl Acad Sci USA 2005, 102, 18538-18543.

41. Sayers T.J.: Targeting the extrinsic apoptosis signaling pathway for cancer therapy. Cancer Immunol Immunother 2011, 60, 1173-1180.

42. Schreiber H., Wu T.H., Nachman J., Kast W.M.: Immunodominance and tumor escape. Semin Cancer Biol 2002, $12,25-31$

43. Schreiber R.D., Old L.J., Smyth M.J.: Cancer immunoediting: integrating immunity's roles in cancer suppression and promotion. Science 2011, 331, 1565-1570.

44. Shevach E.M., Stephens G.L.: The GITR-GITRL interaction: costimulation or contrasuppression of regulatory activity? Nature Rev Immunol 2006, 6, 613-618.

45. Sica A., Schioppa T., Mantovani A., Allavena P.: Tumourassociated macrophages are a distinct M2 polarised population promoting tumour progression: potential targets of anti-cancer therapy. Eur J Cancer 2006, 42, 717-727.

46. Staveley-O'Carroll K., Sotomayor E., Montgomery J., Borello I., Hwang L., Fein S., Pardoll D., Levitsky H.: Induction of antigenspecific T cell anergy: an early event in the course of tumour progression. Proc Natl Acad Sci USA 1998, 95, 1179-1183.

47. Tiemessen M.M., Jagger A.L., Evans H.G., van Herwijnen M.J., John S., Taams L.S.:CD4+CD25+Foxp3+ regulatory $\mathrm{T}$ cells induce alternative activation of human monocytes/macrophages. Proc Natl Acad Sci USA 2007, 104, 19446-19451.

48. Upadhyay R., Hammerich L., Peng P., Brown B., Merad M., Brody J.D.: Lymphoma: immune evasion strategies. Cancers 2015, 7, 736-762.

49. Wesołowski R., Markovitz J., Carson W.E.: Myeloid derived suppressor cells-a new therapeutic target in the treatment of cancer. J Immunother Cancer 2013, 1, 10 http://www.immuno therapyofcancer.org/content/1/1/10

50. Wolchok J.D., Chan T.A.: Cancer: Antitumour immunity gets a boost. Nature 2014, 515, 496-498.

51. Woo S.R., Turnis M.E., Goldberg M.V., Bankoti J., Selby M., Nirschl C.L., Bettini M.L., Gravano D.M., Vogel P., Liu C.L., Tangsombatvisit S., Grosso J.F., Netto G., Smeltzer M.P., Chaux A., Utz P.J., Workman C.J., Pardoll D. M., Korman A.J., Drake C. G., Vignali D.A.A.: Immune inhibitory molecules LAG-3 and PD-1 synergistically regulate T-cell function to promote tumoral immune escape. Cancer Res 2012, 72, 917.

52. Xiao-Feng Qin F.: Dynamic behavior and function of Foxp3 regulatory $\mathrm{T}$ cells in tumor bearing host. Cell Mol Immunol 2009, 6, 3-13.

53. Yang Y.: Cancer immunotherapy: harnessing the immune system to battle cancer. J Clin Invest 2015, 125, 3335-3337.

54. Yang Z.Z., Ansell S.M.: The role of Treg cells in the cancer immunological response. Am J Immunol 2009, 5, 17-28.

55. Yuan A., Hsiao Y.J., Chen H.J., Chen H.W., Ho Ch. Ch., Chen Y.Y., Liu Y.Ch., Hong T.H., Yu S.L., Chen J.J.W., Yang P.Ch.: Opposite effects of M1 and M2 macrophage subtypes on lung cancer progression. Sci Rep 2015, 5, 1-12. 